\title{
Constructal Hypothesis for Mechanical Deformation
}

\author{
Atanu Bikash Chatterjee \\ Dept. of Mechanical Engineering, Bhilai Institute of Technology \\ Indian Astrobiology Research Centre, Mumbai \\ E-mail: achatterjee.jur@gmail.com
}

\begin{abstract}
Mild Steel specimen, when subjected to tensile forces shows considerable plastic deformation before fracture. A cross-section of the fractured specimen has the familiar cup - cone form and shows traces of a three - dimensional parabolic geometry. The morphing of the steel specimen from a volume to a point as a spontaneous, entropy producing or energy dispersing process is analysed using the Constructal law.
\end{abstract}

Keywords: Constructal law, entropy, exergy, mechanical deformation, open system, viscosity.

\section{Introduction}

A Mild Steel specimen that is generally used for tensile testing is an open thermodynamic system as it continuously exchanges mass (corrosion and rusting) and energy with the surrounding media [1]. When an external tensile force is applied, it elongates through a recovery zone to a permanent deformation zone [2]. When the externally applied force per unit area exceeds its maximum stress value, the Ultimate Tensile Strength (UTS), the specimen starts yielding and enters the zone of permanent deformation. Inter - crystalline potential energy prevents the permanent deformation of the specimen in the recovery zone till a critical point or an equilibrium inter - crystalline distance. When externally applied energy exceeds the activation energy or the equilibrium potential energy, yielding of metal starts. Thus, after the critical point, i.e., in the permanent deformation zone, yielding becomes a spontaneous phenomenon. The entropy associated with yielding increases with the elongation of the specimen and is aimed towards 
equalizing energy differences between the surrounding and the excited metallic crystals [3]. Yielding to permanent plastic deformation can be physically characterized by the flow of metal from volume towards a point and thereby causing the cross - sectional area of the specimen to reduce gradually. The morphing of the specimen from a cylindrical solid to a paraboloid is analysed by means of the Constructal law [4].

Constructal law by Bejan, stands today as one of the most fundamental laws of thermodynamics connecting the classical aspects of it, to design generation in nature. According to this law, a system which is free to morph will with time, develop a flow architecture that will give better access to the exergy currents flowing through it $[4,5]$. Flow will arise in a system, if and only if, a gradient of some physical quantity is present within the system or between the system and the surrounding, e.g., diffusion through concentration gradient [6], heat transfer through temperature gradient [7], work transfer through energy gradient, etc. In order to minimize the gradient, energy dispersal will take place with metal flow thus, resulting in an increase in entropy along with increase in length. The elongation process becomes thermodynamically feasible as soon as the energy threshold is reached. The entire work done on the specimen to start sliding is gradually dispersed as heat, out to the surrounding environment hence, causing the rise in temperature as an observable parameter at the time of fracture [8]. At this stage, the metal enters into a plastic state and starts flowing but, inter - planar friction prevents the sliding of one layer of crystal plane over the other. The metal thus behaves as a viscous fluid in motion. We argue here that, the Constructal law generates the flow but, the viscous frictional force along the transverse direction of the flow, imposes the boundary conditions to the flow. Thus, Constructal law supplements and is also, complemented by the viscous force to generate the design topology during cup - cone formation.

\section{Generation of design}

We summarise our ideas as follows:

1) The work done in elongation causes the energy of the metallic crystals to increase and an exergy gradient between respective planes to set up. According to Constructal law, the metal specimen starts to morph in order to give better access to flow of the exergy current.

2) The exergy flow also increases the entropy of the system, making the flow process thermodynamically feasible and gradually dispersing energy to the surrounding environment.

Let us constrain ourselves to the metallic flow/deformation along a two dimensional plane, the $\mathrm{X}-\mathrm{Y}$ plane. The variation in configurational entropy ' $S$ ' 
with change in length at a particular temperature ' $T$ ' hence, assuming isothermal process is represented by the equation [7],

$$
F=T\left(\frac{\partial S}{\partial x}\right)_{T} .
$$

In the above equation, ' $F$ ' is the externally applied force and ' $x$ ' is the varying length along the direction of force. The plastic behaviour is modelled using the Newton's law of viscosity [9]. According to which,

$$
F=\mu A\left(\frac{\partial v}{\partial y}\right) .
$$

In the above equation, ' $\mu$ ' is the viscosity coefficient, ' $A$ ' is the cross- sectional area, ' $v$ ' is the velocity of flow along the direction of applied force and ' $y$ ' is the transverse varying direction. From the above equations it is clear that, entropy increases along the direction of flow or the direction of velocity whereas, viscous force acts along the transverse direction of velocity thus, imposing physical constraints to the flow. The equilibrium point or the design origination point is the point where, both the forces balance each other and thus, converge the flow from a volume to a point. By equating eqn. (1) and (2) we get,

$$
F=T\left(\frac{\partial S}{\partial x}\right)_{T}=\mu A\left(\frac{\partial v}{\partial y}\right) .
$$

Rearranging the above equation we are left with,

$$
T\left(\frac{\partial S}{\partial x}\right)_{T}=\mu A\left(\frac{\partial v}{\partial y}\right)=\frac{T}{\mu A}\left(\frac{\partial S}{\partial t}\right) d y=\left(\frac{d x}{d t}\right) d v .
$$

On integrating, the above equation gives,

$$
\frac{T}{\mu A}\left(\frac{\partial S}{\partial t}\right) \int d y=\int\left(\frac{d x}{d t}\right) d v
$$

Thus we are left with an expression for velocity of flow along $\mathrm{x}-$ axis and viscous force resulting in converging the flow along y - axis. Eqn. (5) when integrated gives an equation of parabolic geometry in a two - dimensional flow of the metal.

$$
y \sim(v(x, t))^{2} .
$$

The above equation thus, governs the generation of cup - cone geometry during tensile testing of a ductile metal specimen. 


\section{Conclusion}

The ideas developed in this paper present a novel approach to investigate the generation of parabolic geometry during the elongation of ductile metallic specimen. The use of Constructal law to understand mechanical deformation shows the hidden unity of all natural laws. The unity is due to the universality of the laws of thermodynamics. Entropy when viewed as an energy dispersing mechanism brilliantly unites the constructal law to mechanical deformation and visco - elastic behavior of metal. Thus, the two forces acting, mutually perpendicular to each other converge the volumetric flow of metal towards a singular point thus, giving rise to a paraboloid of revolution. This is what we usually see when the elastic fracture of the test piece occurs.

\section{Acknowledgement}

I wish to acknowledge the enlightening discussions that I had with dr. dr. Gerard Jagers op Akkerhuis, Senior Researcher, Alterra (The Netherlands) on entropy and Constructal Theory.

\section{References}

[1] P. K. Nag, Engineering Thermodynamics, Tata McGraw Hill Publications, New Delhi, 2008.

[2] S. P. Timoshenko and D. H. Young, Elements of Strength of Materials, Litton Educational Publishing Inc., New York, 2009.

[3] Information on http://www.2ndlaw.oxy.edu/

[4] A. Bejan, Advanced Engineering Thermodynamics, John Wiley, USA, 1998.

[5] A. B. Chatterjee, "Emergence of structures and forms in complex adaptive systems in nature", Sixth International Conference on Relating Design in Nature with Engineering and Science, Spain, 2012.

[6] P. W. Atkins and J. de Paula, Physical Chemistry, Oxford University Press, New York, 2005.

[7] V. Raghavan, Materials Science and Engineering, PHI Learning Private Limited, India, 2004.

[8] W. D. Callister, Jr., Callister's Material Science and Engineering, John Wiley and Sons, 2007.

[9] S. K. Som and G. Biswas, Introduction to Fluid Mechanics and Fluid Machines, Tata McGraw Hill Publications, New Delhi, 2010. 